\title{
Short-term impacts of floods on enteric infectious disease in Qingdao, China, 2005-2011
}

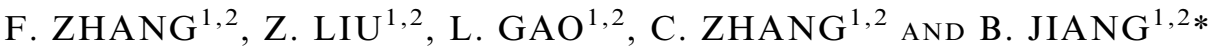 \\ ${ }^{1}$ Department of Epidemiology, School of Public Health, Shandong University, Jinan, Shandong Province, China \\ ${ }^{2}$ Shandong University Climate Change and Health Centre, Jinan, Shandong Province, China
}

Received 12 October 2015; Final revision 16 April 2016; Accepted 6 May 2016;

first published online 17 June 2016

\section{SUMMARY}

The current study aimed to examine the relationship between floods and the three enteric infectious diseases, namely bacillary dysentery (BD), hand-foot-mouth disease (HFMD) and other infectious diarrhoea (OID) in Qingdao, China. Relative risks (RRs) and 95\% confidence intervals (CIs) of floods on BD, HFMD and OID were calculated using a quasi-Poisson generalized linear model, adjusting for daily average temperature, daily average relative humidity, and seasonal and long-term temporal trends. Two separate models within two different periods were designed. Model 1 for the summer period showed that floods were positively associated with BD for 4- to 12-day lags, with the greatest effects for 7-day (RR 1.41, 95\% CI 1.22-1.62) and 11day (RR 1.42, 95\% CI 1.22-1.64) lags. Similar findings were found in model 2 for the whole study period for 5- to 12-day lags. However, HFMD and OID were not significantly associated with floods in both models. Results from this study will provide insight into the health risks associated with floods and may help inform public health precautionary measures for such disasters.

Key words: Bacillary dysentery, floods, hand-foot-mouth disease, other infectious diarrhoea, Poisson generalized linear model.

\section{INTRODUCTION}

Natural hazards, including floods, storms, typhoons and earthquakes, have posed great threats to human lives and property in recent decades. Of these, floods are considered to be the most serious and devastating type of natural disaster globally [1, 2]. In 2010, for example, floods affected 178 million people and led to losses of more than US\$40 billion worldwide [3]. The Intergovernmental Panel on Climate Change

\footnotetext{
* Author for correspondence: Dr B. Jiang, Department of Epidemiology, School of Public Health, Shandong University, No. 44 Wenhuaxi Road, 250012, Jinan, China.

(Email: bjiang@sdu.edu.cn)
}

projects that the areas affected by monsoon precipitation will increase over the 21 st century, and that this will cause or amplify flood events [4]. Shandong Province, a coastal area in eastern China, experienced several floods from 2005 to 2011, with hundreds of people killed and millions more affected [5-11].

Floods have immediate and direct impacts on human health during the initiation phase, with people not only drowning but also experiencing heart attacks, various injuries and hypothermia. Further indirect impacts occur as the floodwaters recede, including water-borne infectious diseases, mental health disorders, respiratory diseases and allergies, and these should also be considered as significant effects [2]. The term 'enteric infection' describes a group of 
diseases caused by various bacterial, viral and parasitic organisms. The major infectious agents are mainly transmitted by the faecal-oral route, often via contaminated water. Several studies have shown that exposure to floods can be associated with high contamination of surface- or groundwater sources, with raw sewage flushed out alongside the deluge of flood waters $[12,13]$. Subsequent transmission of enteric pathogens can then occur, with risk factors including inadequate access to potable water for drinking and washing, poor sanitation caused by the disruption of sanitation infrastructure, displacement of people and sustained exposure to a contaminated environment [13]. Post-flood outbreaks of enteric infectious diseases are common in both developing and developed countries. For example, a communitybased case-control study conducted in Indonesia revealed that flooding was a major risk factor for increased paratyphoid prevalence [comparison with community controls: odds ratio (OR) $4 \cdot 52,95 \%$ confidence interval (CI) 1.90-10.73] [14]. In Mozambique, the incidence of endemic diarrhoea increased following flooding in 2000, due to a deterioration in water quality during the event [15]. Another study from UK also found that individuals exposed to floods had a higher risk of gastroenteritis [16].

Although previous studies have found a high risk of enteric infection following floods, there is a lack of systematic research quantifying the association between flooding and infection in China. Qingdao is a coastal city located in the northeast region of Shandong Province. More than $70 \%$ of the total water supply in Qingdao is obtained from local groundwater and surface-water sources [17]. Excessive precipitation during flooding may pollute local drinking-water sources. Therefore, this study aimed to explore the association between floods and enteric infectious disease in Qingdao, Shandong Province, China.

\section{METHODS}

\section{Study area}

Figure 1 shows the geographical location of Qingdao in Shandong Province. Qingdao is located between longitude $119^{\circ} 30^{\prime}-121^{\circ} 00^{\prime} \mathrm{E}$ and latitude $35^{\circ} 35^{\prime}-$ $37^{\circ} 09^{\prime} \mathrm{N}$, and covers an area of $\sim 11282 \mathrm{~km}^{2}$. The registered population was 9.04 million in 2015 . Qingdao has a typical temperate continental monsoon climate, with an annual average temperature $\left(T_{\mathrm{av}}\right)$ of
$12.7{ }^{\circ} \mathrm{C}$ and annual average rainfall of $662.1 \mathrm{~mm}$. There are three water systems in Qingdao: the Dagu River, the Northern Jiaolai River and coastal rivers. The main source of all three systems is rainfall.

\section{Data collection}

\section{Disease surveillance}

Bacillary dysentery (BD), hand-foot-mouth disease (HFMD) and other infectious diarrhoea (OID) are the three predominant notifiable enteric infectious diseases in Qingdao; the other six species of gastrointestinal disease (i.e. cholera, hepatitis A, hepatitis E, typhoid and paratyphoid fever, poliomyelitis, and acute haemorrhagic conjunctivitis) are quite rare [18-20]. Therefore, this study only examined the impact of floods on BD, HFMD and OID.

Daily disease surveillance data on BD, HFMD and OID from 2005 to 2011 in Qingdao were obtained from the Shandong Center for Disease Control and Prevention (CDC). HFMD was made statutorily notifiable in 2008 , therefore the study period for this disease covered only 4 years (2008-2011) [21]. In China, the surveillance system for notifiable infectious diseases is mainly hospital based. Hospitals at the county level or above are equipped with reference laboratories that are capable of carrying out molecular surveillance. These hospitals form the front line of surveillance for the detection and notification of infectious disease outbreaks in China, combined with laboratories at academic institutions. There are currently 39 notifiable infectious diseases in China, which are classified as $\mathrm{A}, \mathrm{B}$ or $\mathrm{C}$ according to their epidemic level and potential population threat [22]. $\mathrm{BD}$ is categorized as a class B notifiable communicable disease, while HFMD and OID are categorized as class C. Class B represents diseases with a high risk of outbreak or that are likely to spread rapidly once an outbreak has occurred, while class $\mathrm{C}$ diseases are epidemiologically less severe during outbreaks [22]. According to the measures for administration of public health emergencies and communicable disease monitoring information reporting, all hospitals and clinics are obliged to report every case of class $\mathrm{B}$ and $\mathrm{C}$ notifiable communicable diseases to their nominated CDC within $24 \mathrm{~h}$ through the Direct Network Report system [23]. A recent study showed that the CDC reports were of high quality, with $99 \cdot 84 \%$ completeness and $92 \cdot 76 \%$ accuracy [24]. The information collected includes the patient's address, 


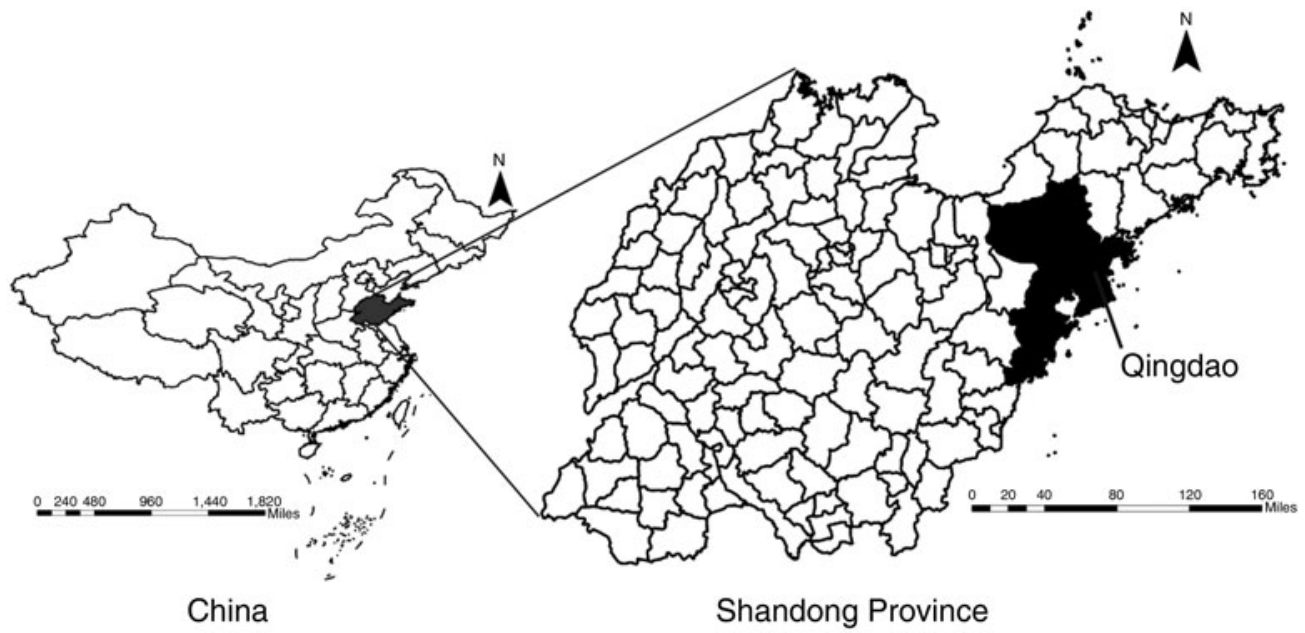

Fig. 1. Location of Qingdao in Shandong Province, China.

reporting unit/hospital, age, sex, name of disease, classification of disease and date of morbidity. We selected patients residing in Qingdao according to their address or the reporting unit/hospital. The date of morbidity was used to calculate the daily case count.

All cases of notifiable communicable diseases were diagnosed according to the unified diagnostic criteria issued by the Chinese Ministry of Health. Only cases that were confirmed clinically and by laboratory tests were included in our study. The diagnostic criteria for the three enteric infectious diseases are as follows:

(1) Clinical diagnosis of $B D$. An enteric infectious disease with fever, stomach-ache, diarrhoea, tenesmus and bloody mucopurulent stool; laboratory diagnosis: cultivation of faeces for Shigella [25].

(2) Clinical diagnosis of HFMD. An infectious gastrointestinal disease with fever, vesicles and sores in the mouth and on the palms, soles and buttocks, with or without neurological abnormalities such as meningitis, encephalitis, and polio-like paralysis [26]; laboratory diagnosis: with enterovirus infection (including EV71, CAV16 or other non-EV71 and non-CAV16 enteroviruses) detected by reverse transcriptase-polymerase chain reaction (RTPCR), real-time RT-PCR, or virus isolation [21].

(3) Clinical diagnosis of OID. A group of diseases caused by a variety of causative agents, such as rotavirus, Salmonella, Vibrio parahaemolyticus and Escherichia coli, and excluding Vibrio cholerae, Shigella and Amoeba, as well as Salmonella typhi and Salmonella paratyphi, with typical clinical symptoms of watery, bloody or mucus stool on more than three occasions per day, with or without dehydration, shock and haemolytic uraemic syndrome [27]; laboratory diagnosis: cultivation of faeces for relevant pathogens.

\section{Flood events}

Data on floods from 2005 to 2011 were collected from the Yearbooks of Meteorological Disasters in China [5-11]. The extent and intensity of floods, as well as the damage to the population, crops and economy ascribed to one specific event, are approximately described in the book. According to the Comprehensive Study Group of Major Natural Disasters of the State Science and Technology Commission in China, a flood is an overflow of surface runoff that submerges towns and farmland [12]. Qingdao experienced 18 floods from 2005 to 2011, which affected hundreds of hectares of crops (Table 1). Because all of the floods were described as city-wide and no information was provided about the specific county or towns affected, all areas of the city were considered to be exposed when a specific flood occurred.

\section{Meteorological data}

Daily meteorological data from 2005 to 2011 were obtained from the China Meteorological Data Sharing Service System (http://cdc.nmic.cn/home.do). Meteorological variables included daily $T_{\mathrm{av}}$, daily average relative humidity $\left(\mathrm{RH}_{\mathrm{av}}\right)$, and daily rainfall.

\section{Statistical analysis}

We examined the short-term effects of floods on three enteric infectious diseases within two different periods: 
Table 1. Description of 18 floods from 2005 to 2011 in Qingdao

\begin{tabular}{lllll}
\hline \hline $\begin{array}{l}\text { Flood } \\
\text { no. }\end{array}$ & Year & Date & $\begin{array}{l}\text { Duration } \\
\text { (days) }\end{array}$ & $\begin{array}{l}\text { Rainfall } \\
(\mathrm{mm})\end{array}$ \\
\hline 1 & 2005 & 3-5 Aug. & 3 & $50-100$ \\
2 & 2005 & 7-9 Aug. & 3 & $50-150$ \\
3 & 2005 & 12-13 Sept. & 2 & $50-100$ \\
4 & 2005 & 19-21 Sept. & 3 & $50-100$ \\
5 & 2005 & 28-30 Sept. & 3 & $50-100$ \\
6 & 2006 & 25-26 Aug. & 2 & 440 \\
7 & 2007 & 27 June & 1 & 150 \\
8 & 2007 & 19 July & 1 & $50-100$ \\
9 & 2007 & 10-12 Aug. & 3 & $50-250$ \\
10 & 2007 & 19-20 Sept. & 2 & $50-200$ \\
11 & 2008 & 18-20 July & 3 & $50-200$ \\
12 & 2008 & 23-24 July & 2 & $50-200$ \\
13 & 2008 & 31 Aug. & 1 & $50-100$ \\
14 & 2009 & 9 July & 1 & $50-100$ \\
15 & 2009 & 12-14 July & 3 & $50-100$ \\
16 & 2010 & 2 July & 1 & $50-100$ \\
17 & 2010 & 26-28 Aug. & 3 & $50-100$ \\
18 & 2011 & 2-3 July & 2 & $50-150$ \\
\hline \hline
\end{tabular}

summer (April-September) and the whole study period from 2005 to 2011. The reason we chose summer was that all floods and nearly $90 \%$ of enteric infection cases occurred during this period. Subsequent analysis through the entire year was performed to verify our results.

We first performed descriptive analyses of the distribution of daily cases and meteorological factors during both periods. Second, relative risks (RRs) and 95\% CIs of the flood effects on BD, HFMD and OID were derived by using a quasi-Poisson generalized linear model to allow for over-dispersion in the case count. Meteorological variables that might influence the incidence of enteric infectious diseases were included in the model. Several studies have explored the associations between diarrhoeal diseases and climate variation such as temperature, rainfall and relative humidity. These variables could directly affect the rate of replication of bacterial and viral pathogens, and the survival of such pathogens in the environment $[28,29]$. Because of the collinearity between flood and daily rainfall, only $T_{\mathrm{av}}$ and $\mathrm{RH}_{\mathrm{av}}$ were adjusted, using two smooth functions of natural spline with three degrees of freedom (D.F.).

We designed two models for the two periods: model 1 for summer and model 2 for the whole study period from 2005 to 2011. In the raw data, long-term patterns including seasonality were likely to dominate the daily case count. As our interest was in short-term associations, the aim was to remove (i.e. control for) these long-term patterns, and to see whether the exposure of interest (i.e. flood) could explain some of the remaining short-term variation. One possible strategy to control for long-term patterns is to fit a spline function of time [30]. In model 1, two natural splines for the day of the year (D.F. $=4$ ) and time (D.F. $=3$ ) were incorporated to control for seasonality and long-term trends [28]. In model 2, seasonality and long-term trends were controlled by a natural spline for time (7 D.F. per year) [31]. The week effect was controlled by an indicator of the day of the week, with a reference of Sunday in both models. Akaike's Information Criterion and analysis of residuals were used to assess the model fit, and to check the autocorrelation of the models. Considering the incubation period of enteric infectious diseases, a lagged effect up to 14 days was assessed [32]. The regression models were described as follows:

Model 1

$$
\begin{aligned}
\log \left[E\left(Y_{t}\right)\right]= & \beta_{0}+\beta_{1}\left(\text { floodlag }_{1-14}\right) \\
& +\operatorname{ns}\left(T_{\mathrm{av}_{1-14}} \operatorname{lag}_{1-1} \text { D.F. }=3\right) \\
& +\mathrm{ns}\left(\mathrm{RH}_{\mathrm{av}} \operatorname{lag}_{1-14}, \text { D.F. }=3\right) \\
& +\mathrm{ns}(\text { doy, D.F. }=4)+\mathrm{ns}(t, \text { D.F. }=3) \\
& + \text { dow. }
\end{aligned}
$$

Model 2

$$
\begin{aligned}
\log \left[E\left(Y_{t}\right)\right]= & \beta_{0}+\beta_{1}\left(\text { floodlag }_{1-14}\right) \\
& +\operatorname{ns}\left(T_{\text {av }} \operatorname{lag}_{1-14}, \text { D.F. }=3\right) \\
& +\operatorname{ns}\left(\mathrm{RH}_{\mathrm{av}} \operatorname{lag}_{1-14}, \text { D.F. }=3\right) \\
& +\mathrm{ns}(t, \text { D.F. }=7 / \text { year })+\text { dow }
\end{aligned}
$$

where $E\left(Y_{t}\right)$ denotes daily cases of BD, HFMD and OID at time $t$, with $t$ equal to the day from 1 January 2005 to 31 December 2011 (from 1 to 2556); $\beta_{0}$ represents the intercept; floodlag represents flooded and non-flooded days using a dummy variable ( 1 and 0 ); $T_{\text {av }}$ lag and $\mathrm{RH}_{\mathrm{av}}$ lag represent daily $T_{\mathrm{av}}$ and $\mathrm{RH}_{\mathrm{av}}$, respectively; doy represents the day of the year and ns denotes a natural spline function with corresponding D.F..

All statistical analyses were performed using $\mathrm{R}$ v. 3.1.1 (The R Project for Statistical Computing, Vienna, Austria). 

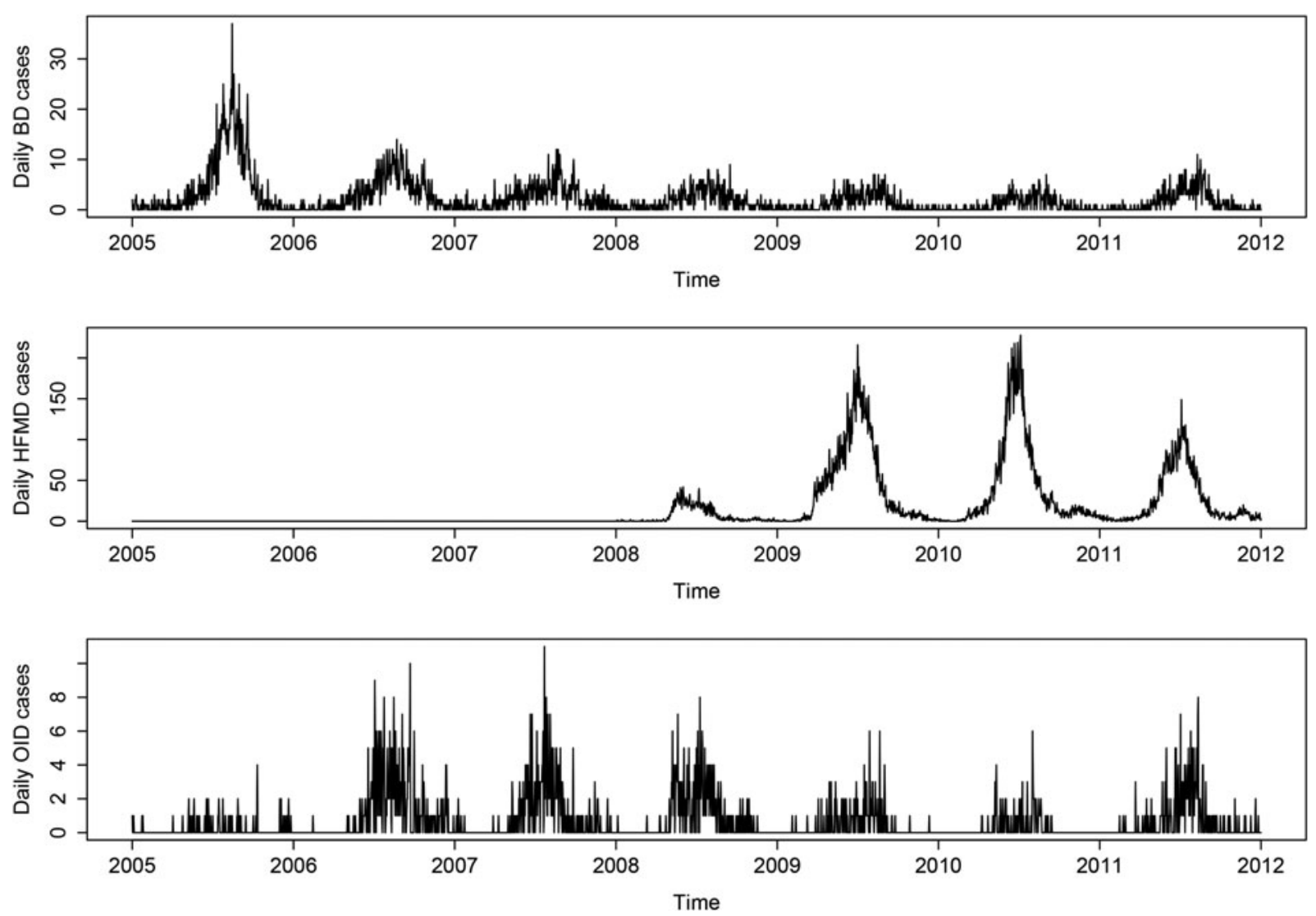

Fig. 2. Daily incidence of enteric infectious disease in Qingdao, 2005-2011. BD, Bacillary dysentery; HFMD, hand-foot-mouth disease; OID, other infectious diarrhoea.

Table 2. Description of daily enteric infectious diseases and meteorological factors during two periods in Qingdao, $2005-2011$

\begin{tabular}{|c|c|c|c|c|c|c|c|c|c|c|c|c|}
\hline \multirow[b]{2}{*}{ Variable } & \multicolumn{6}{|l|}{ Summer } & \multicolumn{6}{|c|}{ Whole study period } \\
\hline & Mean \pm s.D. & Min & $\mathrm{P}_{25}$ & Median & $\mathrm{P}_{75}$ & $\operatorname{Max}$ & Mean \pm s.D. & Min & $\mathrm{P}_{25}$ & Median & $\mathrm{P}_{75}$ & $\operatorname{Max}$ \\
\hline Daily BD cases & $3 \cdot 76 \pm 4 \cdot 05$ & $0 \cdot 0$ & $1 \cdot 0$ & $3 \cdot 0$ & $5 \cdot 0$ & $37 \cdot 0$ & $2 \cdot 25 \pm 3 \cdot 35$ & $0 \cdot 0$ & $0 \cdot 0$ & $1 \cdot 0$ & $3 \cdot 0$ & $37 \cdot 0$ \\
\hline Daily HFMD cases* & $52 \cdot 72 \pm 49 \cdot 91$ & $0 \cdot 0$ & $15 \cdot 0$ & $33 \cdot 5$ & $79 \cdot 0$ & $228 \cdot 0$ & $28 \cdot 66 \pm 42 \cdot 94$ & $0 \cdot 0$ & $2 \cdot 0$ & $9 \cdot 0$ & $35 \cdot 0$ & $228 \cdot 0$ \\
\hline Daily OID cases & $1 \cdot 08 \pm 1 \cdot 60$ & $0 \cdot 0$ & $0 \cdot 0$ & $0 \cdot 0$ & $2 \cdot 0$ & $11 \cdot 0$ & $0 \cdot 62 \pm 1 \cdot 27$ & $0 \cdot 0$ & $0 \cdot 0$ & $0 \cdot 62$ & $1 \cdot 0$ & $11 \cdot 0$ \\
\hline Rainfall (mm) & $3 \cdot 89 \pm 14 \cdot 48$ & $0 \cdot 0$ & $0 \cdot 0$ & $0 \cdot 0$ & $0 \cdot 4$ & $241 \cdot 2$ & $2 \cdot 23 \pm 10 \cdot 57$ & $0 \cdot 0$ & $0 \cdot 0$ & $0 \cdot 0$ & $0 \cdot 0$ & $241 \cdot 2$ \\
\hline$T_{\mathrm{av}}\left({ }^{\circ} \mathrm{C}\right)$ & $20 \cdot 18 \pm 5 \cdot 19$ & $4 \cdot 7$ & $17 \cdot 0$ & $21 \cdot 5$ & $24 \cdot 3$ & $29 \cdot 0$ & $13 \cdot 16 \pm 9 \cdot 34$ & $-8 \cdot 8$ & $4 \cdot 9$ & $14 \cdot 4$ & $21 \cdot 6$ & $29 \cdot 0$ \\
\hline $\mathrm{RH}_{\mathrm{av}}(\%)$ & $76 \cdot 74 \pm 15 \cdot 85$ & $25 \cdot 0$ & $67 \cdot 0$ & $81 \cdot 0$ & $89 \cdot 0$ & $100 \cdot 0$ & $69 \cdot 76 \pm 17 \cdot 19$ & $22 \cdot 0$ & $56 \cdot 0$ & $71 \cdot 0$ & $84 \cdot 0$ & $100 \cdot 0$ \\
\hline
\end{tabular}

S.D., Standard deviation; Min, minimum; $\mathrm{P}_{25}, 25$ th percentile; $\mathrm{P}_{75}, 75$ th percentile; Max, maximum; $\mathrm{BD}$, bacillary dysentery; HFMD, hand-foot-mouth disease; OID, other infectious diarrhoea; $T_{\mathrm{av}}$, daily average temperature; $\mathrm{RH}_{\mathrm{av}}$, daily average relative humidity.

* From 2008 to 2011.

\section{RESULTS}

\section{Disease characteristics and meteorological data}

Figure 2 shows a distinct seasonal distribution of the three enteric infectious diseases. In summer, a total of 4812 BD cases, 38588 HFMD cases and 1388 OID cases were notified by local hospitals, accounting for
$83 \cdot 7 \%, 92 \cdot 2 \%$ and $88 \cdot 0 \%$, respectively, of the total reported cases throughout the whole period from 2005 to 2011 (from 2008 to 2011 for HFMD). Table 2 shows the distributions of the three enteric infectious diseases and meteorological factors for the two different periods. There were some variations in these variables during the two periods. The number of 
Table 3. Flood parameters on the risk of bacillary dysentery in a generalized linear model during two periods in Qingdao, 2005-2011

\begin{tabular}{|c|c|c|c|c|c|c|c|c|c|c|}
\hline \multirow[b]{2}{*}{ Lag (days) } & \multicolumn{5}{|l|}{ Summer } & \multicolumn{5}{|c|}{ Whole study period } \\
\hline & Estimate & S.E. & $z$ & $P$ & $\mathrm{RR}(95 \% \mathrm{CI})$ & Estimate & S.E. & $z$ & $P$ & RR $(95 \%$ CI) \\
\hline Lag 0 & $0 \cdot 02$ & $0 \cdot 08$ & $0 \cdot 23$ & $0 \cdot 821$ & $1 \cdot 02(0 \cdot 87-1 \cdot 19)$ & $-0 \cdot 09$ & $0 \cdot 07$ & $-1 \cdot 30$ & $0 \cdot 194$ & $0 \cdot 91(0 \cdot 79-1 \cdot 05)$ \\
\hline Lag 1 & $-0 \cdot 04$ & $0 \cdot 08$ & $-0 \cdot 51$ & 0.608 & $0.96(0 \cdot 82-1 \cdot 13)$ & $-0 \cdot 15$ & $0 \cdot 07$ & $-2 \cdot 06$ & $0 \cdot 039$ & $0.86(0.75-0.99)$ \\
\hline Lag 2 & $-0 \cdot 10$ & $0 \cdot 09$ & $-1 \cdot 21$ & $0 \cdot 227$ & $0.90(0 \cdot 76-1 \cdot 07)$ & $-0 \cdot 21$ & $0 \cdot 07$ & $-2 \cdot 86$ & $0 \cdot 004$ & $0 \cdot 81(0 \cdot 70-0 \cdot 94)$ \\
\hline Lag 3 & $0 \cdot 06$ & $0 \cdot 08$ & $0 \cdot 73$ & $0 \cdot 467$ & $1.06(0.91-1 \cdot 24)$ & $-0 \cdot 06$ & $0 \cdot 07$ & $-0 \cdot 80$ & $0 \cdot 424$ & $0.95(0.82-1 \cdot 08)$ \\
\hline Lag 4 & $0 \cdot 22$ & $0 \cdot 08$ & $2 \cdot 83$ & $0 \cdot 005$ & $1 \cdot 24(1 \cdot 07-1 \cdot 45)$ & $0 \cdot 10$ & $0 \cdot 07$ & $1 \cdot 54$ & $0 \cdot 123$ & $1 \cdot 11(0.97-1 \cdot 27)$ \\
\hline Lag 5 & $0 \cdot 23$ & $0 \cdot 08$ & $3 \cdot 05$ & $0 \cdot 002$ & $1 \cdot 26(1 \cdot 09-1 \cdot 47)$ & $0 \cdot 14$ & $0 \cdot 07$ & $2 \cdot 04$ & $0 \cdot 042$ & $1 \cdot 15(1 \cdot 01-1 \cdot 30)$ \\
\hline Lag 6 & $0 \cdot 33$ & $0 \cdot 07$ & $4 \cdot 45$ & $0 \cdot 000$ & $1 \cdot 39(1 \cdot 20-1 \cdot 60)$ & $0 \cdot 24$ & $0 \cdot 06$ & $3 \cdot 67$ & $0 \cdot 000$ & $1 \cdot 27(1 \cdot 12-1 \cdot 44)$ \\
\hline Lag 7 & $0 \cdot 34$ & $0 \cdot 07$ & $4 \cdot 69$ & $0 \cdot 000$ & $1 \cdot 41(1 \cdot 22-1 \cdot 62)$ & $0 \cdot 26$ & $0 \cdot 06$ & $4 \cdot 00$ & $0 \cdot 000$ & $1 \cdot 29(1 \cdot 14-1 \cdot 46)$ \\
\hline Lag 8 & $0 \cdot 31$ & $0 \cdot 07$ & $4 \cdot 11$ & $0 \cdot 000$ & $1 \cdot 36(1 \cdot 17-1 \cdot 57)$ & $0 \cdot 20$ & $0 \cdot 07$ & $3 \cdot 08$ & $0 \cdot 002$ & $1 \cdot 23(1.08-1 \cdot 39)$ \\
\hline Lag 9 & $0 \cdot 28$ & $0 \cdot 08$ & $3 \cdot 77$ & $0 \cdot 000$ & $1 \cdot 33(1 \cdot 15-1 \cdot 54)$ & $0 \cdot 19$ & $0 \cdot 07$ & $2 \cdot 90$ & $0 \cdot 004$ & $1 \cdot 21(1 \cdot 06-1 \cdot 38)$ \\
\hline Lag 10 & $0 \cdot 32$ & $0 \cdot 07$ & $4 \cdot 28$ & $0 \cdot 000$ & $1 \cdot 38(1 \cdot 19-1 \cdot 60)$ & $0 \cdot 22$ & $0 \cdot 07$ & $3 \cdot 39$ & $0 \cdot 001$ & $1 \cdot 25(1 \cdot 10-1 \cdot 42)$ \\
\hline Lag 11 & $0 \cdot 35$ & $0 \cdot 08$ & $4 \cdot 61$ & $0 \cdot 000$ & $1 \cdot 42(1 \cdot 22-1 \cdot 64)$ & $0 \cdot 25$ & $0 \cdot 07$ & $3 \cdot 78$ & $0 \cdot 000$ & $1 \cdot 28(1 \cdot 13-1 \cdot 46)$ \\
\hline Lag 12 & $0 \cdot 25$ & $0 \cdot 08$ & $3 \cdot 09$ & $0 \cdot 002$ & $1 \cdot 28(1 \cdot 10-1 \cdot 50)$ & $0 \cdot 17$ & $0 \cdot 07$ & $2 \cdot 43$ & $0 \cdot 015$ & $1 \cdot 18(1 \cdot 03-1 \cdot 35)$ \\
\hline Lag 13 & $0 \cdot 07$ & $0 \cdot 09$ & $0 \cdot 81$ & $0 \cdot 419$ & $1 \cdot 07(0 \cdot 90-1 \cdot 27)$ & $0 \cdot 00$ & $0 \cdot 07$ & $0 \cdot 07$ & 0.947 & $1 \cdot 00(0 \cdot 87-1 \cdot 16)$ \\
\hline Lag 14 & $0 \cdot 09$ & $0 \cdot 09$ & $1 \cdot 00$ & $0 \cdot 316$ & $1.09(0.92-1 \cdot 30)$ & $0 \cdot 03$ & $0 \cdot 07$ & $0 \cdot 48$ & 0.634 & $1 \cdot 04(0 \cdot 90-1 \cdot 19)$ \\
\hline
\end{tabular}

RR, Relative risk; CI, confidence interval.

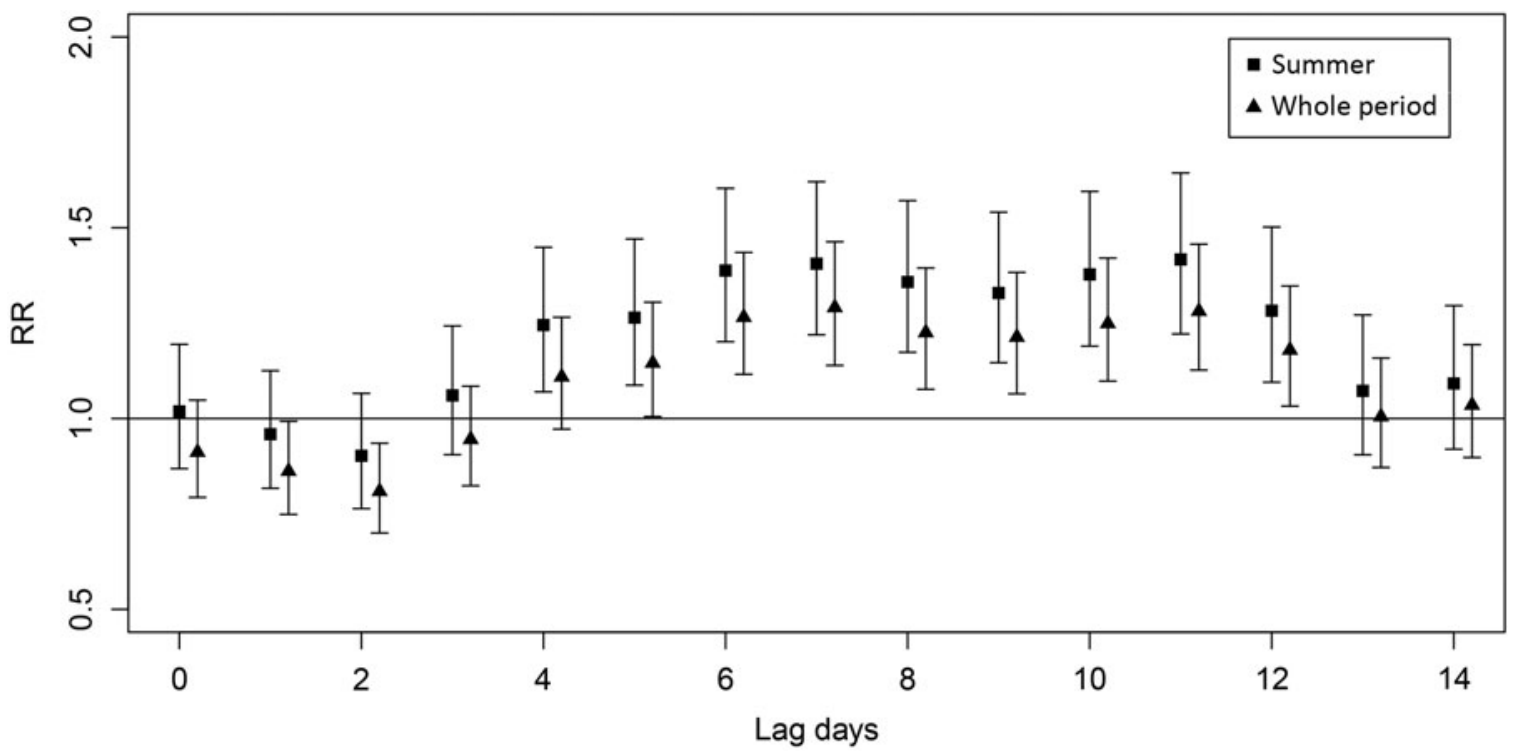

Fig. 3. Relative risk (RR) estimates of floods on bacillary dysentery at different lagged days within two periods in Qingdao, 2005-2011.

cases of daily enteric infectious diseases and all meteorological factors were higher during summer days than over the whole study period.

\section{Regression analysis using a generalized linear model at different time lags}

Table 3 and Figures 3-5 show the estimated RRs of floods on the three enteric infectious diseases for 1- to 14-day lags. The results shown in Table 3 and
Figure 3 indicate broadly consistent increased risks for BD during floods across the two periods (summer: RR > 1 for 4- to 12-day lags; whole study period: $\mathrm{RR}>1$ for 5- to 12-day lags), while the two strongest lagged effects in model 1 for summer were slightly larger than those in model 2 for the whole study period (model 1, 7-day lag: RR 1.41, 95\% CI 1.22-1.62; 11-day lag: RR $1 \cdot 42$, 95\% CI $1 \cdot 22-1 \cdot 64$; model 2, 7-day lag: RR $1 \cdot 29$, 95\% CI $1 \cdot 14-1 \cdot 46$; 11-day lag: RR 1.28, 95\% CI 1·13-1·46). HFMD 


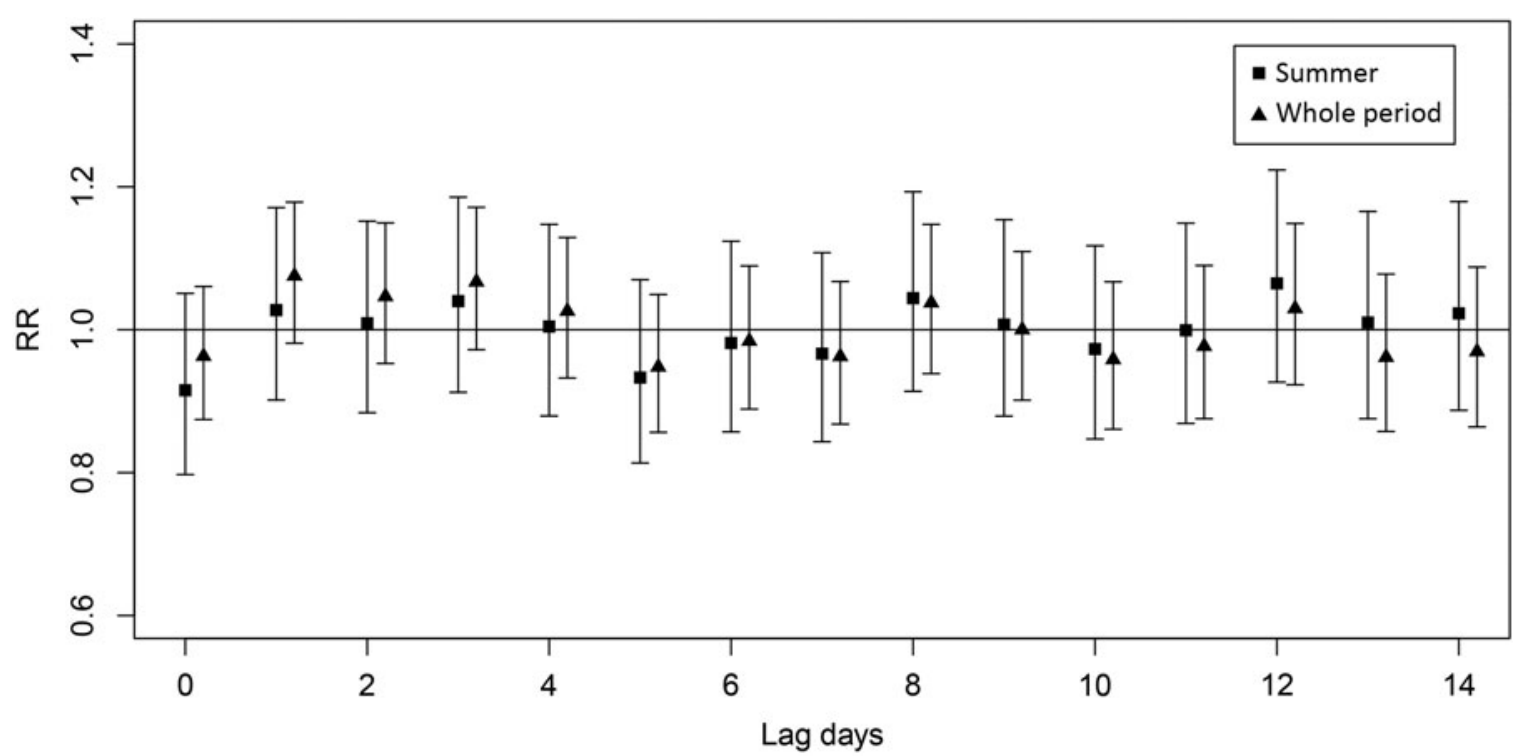

Fig. 4. Relative risk (RR) estimates of floods on hand-foot-mouth disease at different lagged days within two periods in Qingdao, 2005-2011.

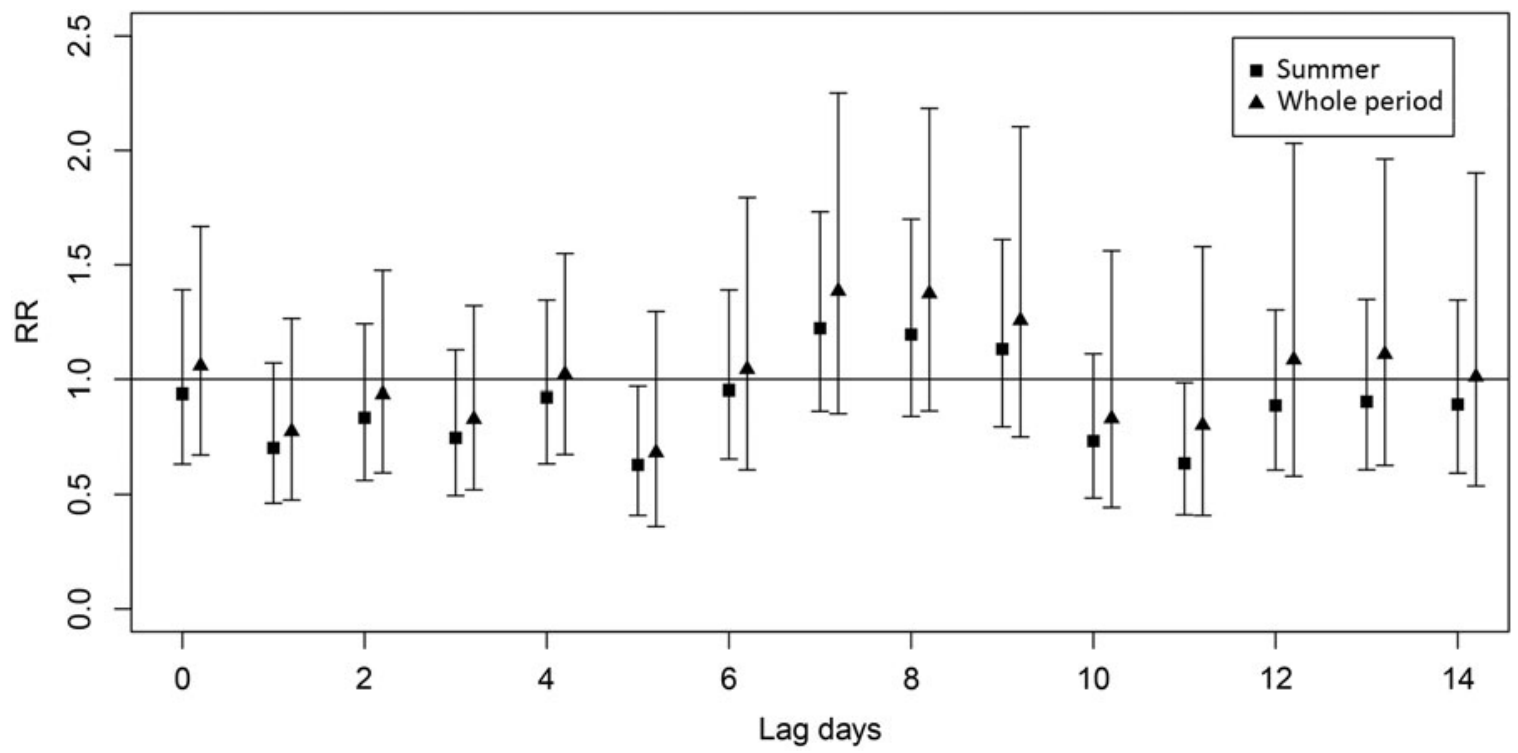

Fig. 5. Relative risk (RR) estimates of floods on other infectious diarrhoea at different lagged days within two periods in Qingdao, 2005-2011.

and OID were not significantly associated with floods (Figs 4 and 5).

\section{DISCUSSION}

In recent years, under the context of climate change, enteric infectious diseases are increasingly being recognized as a significant health problem. This study stands out from previous research by highlighting the critical role of floods in driving the transmission of $\mathrm{BD}$. Our results indicate that floods are positively associated with the incidence of $\mathrm{BD}$ in the selected study area, while no significant flood effects were found for HFMD or OID. Results from this study will have significant implications for developing strategies to prevent and reduce the health impacts of floods.

$\mathrm{BD}$ is an enteric infectious disease that is transmitted through the faecal-oral route via contaminated water, food, daily contact and flies [25]. In China, $\mathrm{BD}$ is still an important public health problem, and 
has caused more than 300 deaths per year for the last decade [29]. Cases of BD usually occur in areas that are crowded and have poor sanitary conditions [28, 29, 33, 34]. However, limited studies have examined the effects of floods on BD and the results have varied across different studies. In the present study, we found that floods are associated with an increase in daily BD cases, echoing the study of $\mathrm{Ni}$ et al. [35]. Using longitudinal data from 2004 to 2009, Ni et al. examined the relationship between monthly dysentery cases and floods in three cities of Henan Province, and found higher morbidity from dysentery during flooded compared to non-flooded months (RR 1.66, 95\% CI 1.521.82) [35]. There are several possible pathways by which floods might affect BD incidence. For example, excessive rainfall can move pathogens in the environment into rivers, coastal waters and wells. This can adversely affect water sources and supply systems, and thereby accelerate the transmission of enteric pathogens [36]. In urban areas, if the wastewater volume in a combined sewerage system exceeds the capacity of the sewerage system or treatment plant, then excess wastewater, containing not only storm water but also untreated human waste, can directly flow into nearby streams, rivers and other water bodies, and therefore pollute drinking water in these areas [36]. In addition, secondary effects of flooding, namely high population densities and forced migration from affected homes or workplaces in high-risk areas, might also contribute to the spread of BD [4].

The current study found that floods are positively associated with $\mathrm{BD}$ from 4- to 12-day lags, with the greatest effects at 7-day (RR 1.41, 95\% CI 1.221.62) and 11-day (RR 1.42, 95\% CI 1.22-1.64) lags. Although the incubation period of $\mathrm{BD}$ is 7 days, we believe that the positive effect at longer time lags is more biologically plausible, considering the time required for the pathogen to pollute water or food.

A positive relationship between floods and HFMD was not detected in this study. HFMD epidemics have been reported worldwide in recent decades, particularly in the Asia-Pacific region [24]. In mainland China, numerous HFMD outbreaks have recently been reported [21]. For example, in 2010, more than one million HFMD cases, resulting in 509 deaths, were reported [24]. To the best of our knowledge, the association between floods and HFMD has not previously been studied. However, the relationship between extreme precipitation and HFMD has been investigated in several studies, with inconsistent results. Cheng et al. reported a significantly positive association between extreme precipitation and childhood HFMD in Hefei, China [37]. In contrast, Hii et al. reported a negative correlation, with each millimetre increase in rainfall decreasing the HFMD incidence by $0.5 \%$ (95\% CI 0.995-0.996) when the weekly cumulative precipitation was more than $75 \mathrm{~mm}$ in Singapore [38]. Moreover, Onozuka \& Hashizume observed no relationship between precipitation and HFMD in Japan [39]. There is no clear explanation for such inconsistencies, which might be partially attributed to a threshold effect of precipitation on HFMD. It is possible that people living in coastal China and island countries such as Singapore, where there is high precipitation and humidity, might have a relatively higher adaptive capacity to extreme precipitation than those living in temperate cities [37]. Another potential explanation relates to the route of transmission of HFMD; aside from contact with contaminated environments such as water, food and surface, HFMD is mainly transmitted through direct contact with respiratory droplets and the blister fluid of infectious patients.

The incidence of OID is also high in China. In 2011, 836591 cases of OID were reported, with an incidence rate of $62 \cdot 39 / 100000$ population. Our study did not find a positive relationship between floods and OID, even though $88.0 \%$ of OID cases occurred during the peak flooding period (April-September) when the weather was rainy. The underlying mechanism behind this phenomenon remains unclear, and requires further study.

One advantage of our study is that we applied a generalized linear model to investigate the day-by-day impact of floods on three enteric disease in Qingdao, Shandong Province, allowing us to flexibly examine the possible relationships between weather conditions and disease. A previous study suggested that a day-by-day analysis is the best approach for diseases with a short incubation period, and that such results would be of greatest benefit for disease control and prevention by providing timely information [40].

Several limitations of this study must be acknowledged. First, information about the specific living conditions of patients, household size, family income, parents' literacy, availability of health services and individual behaviours were not available from our data sources. In addition, under-reporting is an inevitable issue, which could lead to an underestimation of the risk of enteric infectious diseases attributed to floods. Last, all floods were described as city-wide, which might also lead to an underestimated RR value if some towns were not affected by the flooding. 


\section{CONCLUSIONS}

In conclusion, our study has confirmed that floods can significantly increase the risk of BD in the study area, while no positive flood effects on HFMD and OID were detected. Results from this study will provide insight into the health risks associated with floods and may help inform public health precautionary measures for such disasters.

\section{ACKNOWLEDGEMENTS}

We are extremely grateful to the Shandong Center for Disease Control and Prevention and the National Meteorological Information Centre of China for sharing the data needed for the study.

This work was supported by the National Basic Research Program of China (973 Program) (grant no. 2012CB955502).

\section{DECLARATION OF INTEREST}

None.

\section{REFERENCES}

1. Howard MJ, Brillman JC, Burkle Jr. FM. Infectious disease emergencies in disasters. Emergency Medicine Clinics of North America 1996; 14: 413-428.

2. Messeri A, et al. Weather-related flood and landslide damage: a risk index for Italian Regions. PLoS ONE 2015; 10: e0144468.

3. Mogollon B, et al. An empirical assessment of which inland floods can be managed. Journal of Environmental Management 2016; 167: 38-48.

4. Patz JA, Grabow ML, Limaye VS. When it rains, it pours: future climate extremes and health. Annals of Global Health 2014; 80: 332-344.

5. Dong WJ. Yearbook of Meterorological Disasters in China, 1st edn [in Chinese]. Beijing: China Meteorological Press, 2006, pp. 20.

6. Dong WJ. Yearbook of Meterorological Disasters in China, 1st edn [in Chinese]. Beijing: China Meteorological Press, 2007, pp. 23.

7. Xiao ZN. Yearbook of Meterorological Disasters in China, 1st edn [in Chinese]. Beijing: China Meteorological Press, 2008, pp. 19.

8. Xiao ZN. Yearbook of Meterorological Disasters in China, 1st edn [in Chinese]. Beijing: China Meteorological Press, 2009, pp. 18.

9. Song LC. Yearbook of Meterorological Disasters in China, 1st edn [in Chinese]. Beijing: China Meteorological Press, 2010 , pp. 22.

10. Song LC. Yearbook of Meterorological Disasters in China, 1st edn [in Chinese]. Beijing: China Meteorological Press, 2011, pp. 17.
11. Song LC. Yearbook of Meterorological Disasters in China, 1st edn [in Chinese]. Beijing: China Meteorological Press, 2012, pp. 18.

12. Ding G, et al. Quantitative analysis of burden of infectious diarrhea associated with floods in northwest of Anhui province, China: a mixed method evaluation. PLoS ONE 2013; 8: e65112.

13. Ahern M, et al. Global health impacts of floods: epidemiologic evidence. Epidemiologic Reviews 2005; 27: 36- 46.

14. Vollaard AM, et al. Risk factors for typhoid and paratyphoid fever in Jakarta, Indonesia. Journal of the American Medical Association 2004; 291: 2607-2615.

15. Kondo H, et al. Post-flood-infectious diseases in Mozambique. Prehospital and Disaster Medicine 2002; 17: 126-133.

16. Reacher M, et al. Health impacts of flooding in Lewes: a comparison of reported gastrointestinal and other illness and mental health in flooded and non-flooded households. Communicable Disease and Public Health 2004; 7: 39-46.

17. Li Y, et al. Life cycle assessment of water supply alternatives in water-receiving areas of the South-to-North Water Diversion Project in China. Water Research 2015; 89: 9-19.

18. Gong $\mathbf{X Y}, \mathbf{W u} \mathbf{W}$. The epidemiological characteristics of infectious diarrhea caused by agents other than vibrio cholerae, salmonella and shigella in Laiyang city, Shandong province, 2009 [in Chinese]. China Preventive Medicine 2010; 8: 795-796.

19. Xu LS, Li YL, Fan AP. Incidences of notifiable communicable diseases of Taian city in Shandong province, 2010 [in Chinese]. Disease Surveillance 2011; 8: 639-642.

20. Zhang GQ, Wang XQ, Fu ZJ. Epidemiological feature of notifiable infectious diseases in Shouguang City of Shandong Province, 2010-2013 [in Chinese]. Bulletin of Disease Control and Prevention 2014; 3: 13-15.

21. Xing W, et al. Hand, foot, and mouth disease in China, 2008-12: an epidemiological study. Lancet Infectious Diseases 2014; 14: 308-318.

22. Zhang L, Wilson DP. Trends in notifiable infectious diseases in China: implications for surveillance and population health policy. PLoS ONE 2012; 7: e31076.

23. National Health and Family Planning Commission. The measures for administration of public health emergencies and communicable disease monitoring information reporting (No. 37 decree of Ministry of Health), 2006 (http:// www.moh.gov.cn/mohzcfgs/pgz/200901/38689.shtml).

24. Yang H, et al. Is high relative humidity associated with childhood hand, foot, and mouth disease in rural and urban areas? Public Health 2015; Published online: 30 April 2015. doi:10.1016/j.puhe.2015.03.018.

25. Ma W, et al. Applied mixed generalized additive model to assess the effect of temperature on the incidence of bacillary dysentery and its forecast. PLoS ONE 2013; 8: e62122.

26. Song Y, et al. Time series analyses of hand, foot and mouth disease integrating weather variables. PLoS ONE 2015; 10: e0117296.

27. Liu HX, Zhang J. Analysis of reported infectious diarrhea (other than cholera, dysentery, typhoid and paratyphoid) in China in 2011. Chinese Journal of Preventive Medicine 2013; 47: 328-332. 
28. Huang D, et al. Investigating the effects of climate variations on bacillary dysentery incidence in northeast China using ridge regression and hierarchical cluster analysis. BMC Infectious Diseases 2008; 8: 130.

29. Zhang Y, Bi P, Hiller JE. Weather and the transmission of bacillary dysentery in Jinan, northern China: a timeseries analysis. Public Health Reports 2008; 123: 61-66.

30. Bhaskaran $\mathbf{K}$, et al. Time series regression studies in environmental epidemiology. International Journal of Epidemiology 2013; 42: 1187-1195.

31. Gasparrini A. Distributed lag linear and non-linear models for time series data. 2014 (https://cran.r-project. org/web/packages/dlnm/vignettes/dlnmTS.pdf).

32. Yang SJ, Ren H. Lemology, 7th edn. Beijing: People's Medical Publishing House, 1980.

33. Chen T, et al. Investigation of key interventions for shigellosis outbreak control in China. PLoS ONE 2014; 9: e95006.

34. Gao L, et al. Meteorological variables and bacillary dysentery cases in Changsha City, China. American Journal of Tropical Medicine and Hygiene 2014; 90: 697-704.
35. Ni W, et al. Effects of the floods on dysentery in north central region of Henan Province, China from 2004 to 2009. Journal of Infection 2014; 69: 430-439.

36. Cann KF, et al. Extreme water-related weather events and waterborne disease. Epidemiology and Infection 2013; 141: 671-686.

37. Cheng $\mathbf{J}$, et al. Associations between extreme precipitation and childhood hand, foot and mouth disease in urban and rural areas in Hefei, China. Science of the Total Environment 2014; 497-498: 484-490.

38. Hii YL, Rocklov J, Ng N. Short term effects of weather on hand, foot and mouth disease. PLOS ONE 2011; 6: e16796.

39. Onozuka D, Hashizume M. The influence of temperature and humidity on the incidence of hand, foot, and mouth disease in Japan. Science of the Total Environment 2011; 410-411: 119-125.

40. Chen C, et al. Short-term effects of meteorological factors on children hand, foot and mouth disease in Guangzhou, China. International Journal of Biometeorology 2014; 58: $1605-1614$. 